\section{RECORDS OF 100 ADMINISTRATIONS EACH OF CHLORIDE OF ETHYL AND OF SOMNOFORM ALONE AND IN MIXTURE WITH NITROUS OXIDE.}

BY G. W. BAMPFYLDE DANIELL, M.R.C.S. ENG., L.R.C.P. LOND.,

LATE LECTURER ON, AND INSTRUCTOR IN, AN ASTHETICS TO THE ROYAT INFIRMARY, EDINBURGH; LATE ANFSTHETIST TO THE DENTAI. HOSPITAL, EDINBURGH.

AlTHOUGH fully mindful of the important work done by many interested in those anæsthetic agents which of recent years have re-appeared and are now being so largely used, yet there is, I think, a want of a greater number of recorded administrations conducted in a systematic way under such conditions and circumstances as can only be obtained in a large medical institution. It is obvious that so few cases as 400 can be of but little help in forming an opinion, nevertheless their publication may be of interest to some who will add them to their own and to those collected from other sources. As yet it is quite clear that opinions differ widely in relation to certain details in connexion with any one of these anæsthetics and the reasons are not far to seek. It may take years to collect enough trustworthy information before we are able to decide definitely points at issue, and it behoves all those who have the opportunity to add what knowledge they possess in furtherance of this end. In writing this paper my object is to be as brief as possible, confining myself entirely to the 400 cases and only adding such remarks as bear directly on the records.

Conditions under which the records were made.-All the administrations were conducted in the same room-i.e., the extracting-room of the dental department at the Edinburgh Royal Infirmary. The records were commenced in July, 1903, and continued at intervals until March, 1905. The dental surgeons attend every Wednesday mid-day and the work is generally finished in the space of about two hours. With a few exceptions all the cases were taken without selection. Most were of the usual type of patient visiting any of our great hospitals : some were in-patients of the infirmary, some were sent from other hospitals, the consump. tive and lock, for instance ; others were medical practitioners, students, nurses, and hospital servants, so that it will be seen that this list includes many classes of humanity. The greater number of administrations were given by myself, practically all the chloride of ethyl and somnoform alone, and the nitrous oxide and chloride of ethyl mixture. It should be stated that before undertaking these administrations $I$ had given a number in London by various methods and using a variety of inhalers, and had also seen others conduct administrations both in London and Edinburgh. In a few cases the anæsthetic was given by postgraduates or students under my supervision or by one of the dental surgeons, both the latter being skilled anæsthetists as far as the requirements of dental surgery are concerned. The notes and observations on each case were entered shortly after the patient's recovery. This part of the work was mainly done by one whose practical knowledge and experience in dental surgery as well as anæsthetics are beyond dispute.

The inhaler used.-Generally speaking, the inhaler used and the method of administration were the same as described by me in THE LANCET of Oct. 17th, 1903, p. 1087, and in the British Medical Journal of April 23rd, 1904, a brief description of which is as follows. The ether chamber of the ordinary Clover's portable ether inhaler is removed, and the facepiece and the angle-mount (holding the bag of red rubber of one-gallon capacity) are attached. To the anglemount are fitted an extra large metal tap and tube, the latter projecting into the bag. To this tap is attached a small measure of thick glass, which I had specially made with a flat bottom so that it will stand alone, and graduated to show three, four, and five cubic centimetres. A short piece of red rubber tubing connects this glass to the tap, being securely fixed to the former (vide Fig. 1). The following are the points of advantage claimed by this inhaler and method of administration. 1. A measured quantity of the anæsthetic fluid is given, varying according to the age of the patient and taking into consideration the time of anæsthesia required, the nature of the operation, \&c. This gives accuracy of dosage and is useful for making observations and notes for future reference. It should also help to insure against an over. dose. 2. The anæsthetic can be given gradually and that after the facepiece is adjusted; this prevents coughing, holding the breath, \&c., and produces a satisfactory induction. 3. More can at any time be added if necessary,

FIG. 1.

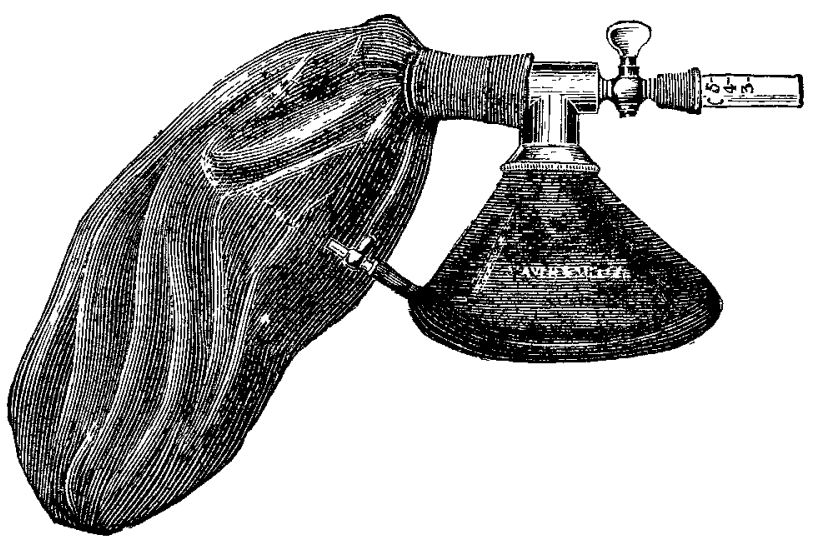

Author's inhaler.

either by projecting the fluid through the large open tap or by measuring off into the tube; also, either can be done without having to remove the facepiece, which is an advantage in some cases in which air-exclusion is necessary. On the other hand, the tube need not be emptied if satisfactory anæsthesia is likely to be produced by less than its contents. In hospital practice this is kept over for the next case for purposes of economy. 4. In the event of voluntary struggling-as is sometimes the case in children and very nervous subjects-it is easy to empty the tube by tipping it up with one finger, both hands being available to hold the mask and to steady the patient. 5. If the tap is turned off before the tube is fixed on the patient will not experience the slightest smell of the narcotic when the mask is applied. This is of special importance in nervous patients. 6. A perfectly free air-way, no lint, sponge or wool to breathe through, and no freezing vapour near the patient's mouth or nose, as all evaporation takes place in the metal tube and rubber bag. 7. Nitrous oxide can be given as a preliminary. 8. By retaining the ether chamber these drugs can be given with ether or used as a sequence; all that is necessary is the glass tube. (Vide Fig. 2.) 9. Economy in the amount of anæsthetic used.

FIG. 2.

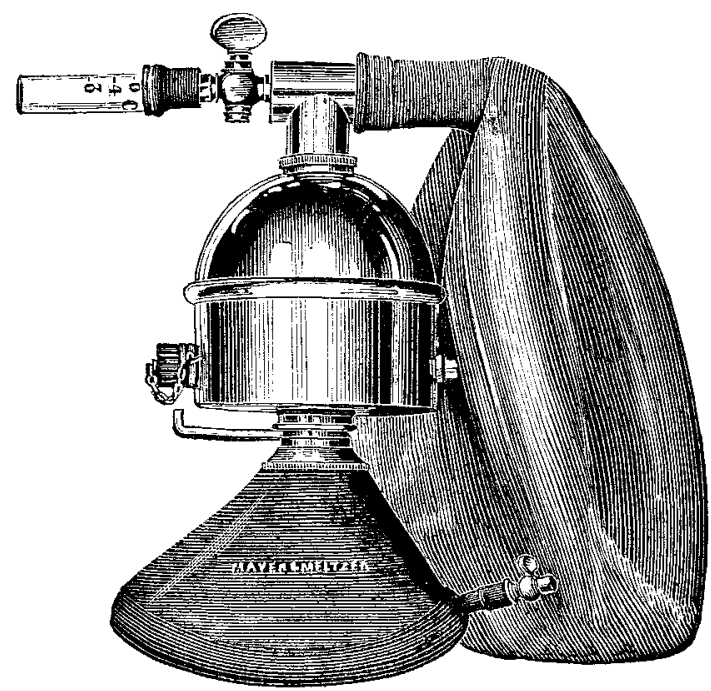

The anæsthetic agent is first measured into the glass tube which is then attached to the closed tap. The mask with the tube pointing downwards is applied at the end of an inspiration and must fit the face accurately. A short period of re-breathing is now permitted, the tap is then opened, and at once the angle-mount is rotated until the glass tube is almost horizontal. By gently elevating the glass measure with one finger the fluid flows slowly out through the tap and tube and into the bag. Part of the fluid will evaporate on passing through the tap and tube as it offers a considerable metallic surface. My object in the administration is to present to the patient a dilute vapour at first until signs 
of unconsciousness begin to show themselves, then the drug is given more rapidly. Coughing or holding the breath is generally taken as an indication of the vapour being rather too strong for that individual case and in most instances the evaporation of the drug is temporarily checked by turning down the angle-mount or depressing the glass tube until the symptoms vanish. Chloride of ethyl and somnoform were both administered in this way. No capsules were used in any case. Various makes of chloride of ethyl were used but chiefly that of Duncan, Flockhart and Co., also Hedley's and that of Bengué. A uniform system of note-taking was adopted, examples of which are given below.

Chloride of Ethyl Alone.

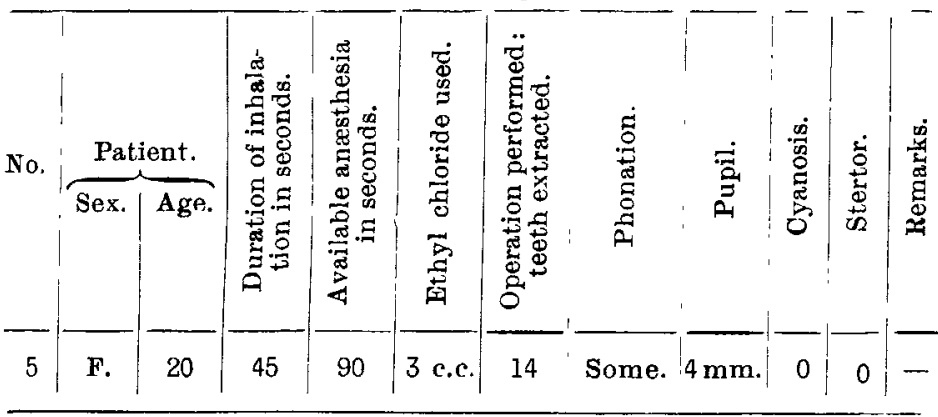

Nitrous Oxide and Somnoform.

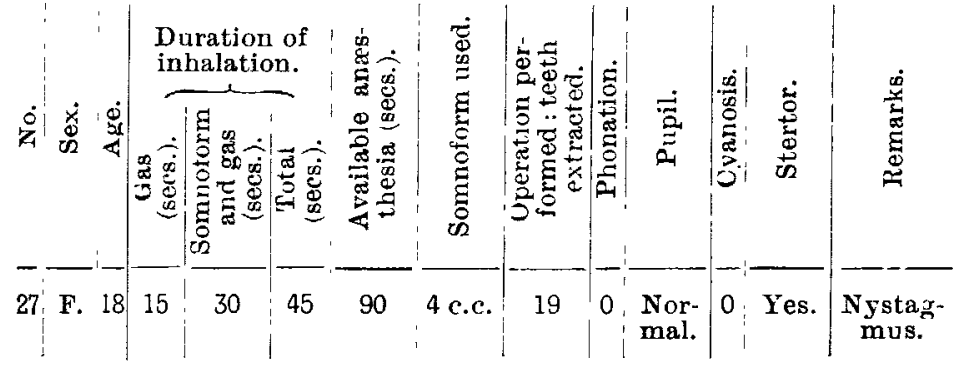

Chloride of ethyl. - Of the 100 cases 80 were females, 19 were males; the sex in one was not recorded. The oldest patient was 52 years and the youngest was eight years. The duration of inhalation or period of induction was counted from the time the patient began to inhale the vapour until the anæsthesia was considered deep enough for the particular amount of operative work to be performed. The average for the hundred was $46 \cdot 65$ seconds. The longest was 105 seconds. This was due largely to the patient voluntarily holding her breath. The shorte:t periods were 30 seconds. Two cases required a second dose. In both of these, for the sake of uniformity, I take the induction period of the first inhalation only. Full notes of these two are as follows. Case 69, a female, aged 18 years. Duration of induction first time, 45 seconds ; second time, 30 seconds. Available anæsthesia, first time, 50 seconds ; second time, 20 seconds. First dose, three cubic centimetres; second dose two cubic centimetres. Two teeth were extracted. There was phonation. The pupils were four millimetres. There was no cyanosis. Stertor was present. The patient was an alcoholic. There were much excitement and struggling and it was necessary to give a second dose, as the operator, a student, had failed to extract the teeth. Case 80 was a female, aged 22 years. Induction period, first dose, 60 seconds; second dose, 30 seconds. Available anæsthesia, first dose, 60 seconds; second, 90 seconds; three cubic centimetres were given each time. Teeth extracted 18. There was no phonation. The pupils were four millimetres. There was no cyanosis or stertor. The anæsthesia was prolonged by a second inhalation as the operation was difficult.

Available ancesthesia.-From the moment the facepiece was removed until some sign of recovery was shown, such as movement, the longest anæsthesia was 205 seconds. Case 66 was a female, aged 31 years. Induction period, 70 seconds. The dose was six cubic centimetres. The teeth extracted numbered 25 . The pupils were five millimetres. There was no cyanosis, phonation, or stertor. This case was very good. The two cases in which re-inhalation was necessary are treated in the same way as under "duration of inhalation." The shortest time was 20 seconds, Case 55, a female, aged 13 years. Induction period, 35 seconds. The dose was three cubic centimetres. 'The teeth extracted numbered two. The pupils were three millimetres. There was no phonation, cyanosis, or stertor. The average anæsthesia was as near as possible 75 seconds.
Amount of ethyl chloride used.-This was generally determined by the amount of operative work necessary in each individual case, taking into consideration the age of the patient, sex, \&c. The largest dose given was six cubic centimetres. There were two cases; one is recorded above; the other was a female, aged 23 jears. Induction took 85 seconds; anæsthesia lasted 85 seconds. The number of teeth extracted was eight. There was phonation. The pupils were four millimetres. There was no cyanosis or stertor. 'There were excitement and speaking on going under. The smallest dose was two cubic centimetres. There were two cases. Case 3 was a female, aged 12 years. Induction period, 30 seconds ; anæsthesia lasted 35 seconds. The number of teeth extracted was four. The pupils were three millimetres. There was no phonation, cyanosis, or stertor. Good anæsthesia. The other case was that of a child, aged five years. The induction period was 50 seconds; anæsthesia lasted 60 seconds. One tooth was extracted. The pupils were normal. There was no cyanosis, \&c. The average dose given in the 100 cases was $3 \cdot 74$ cubic centimetres.

The greatest number of teeth extracted in any one case was 25; next to this 23 ; and then 20 . One in two instances. The average number was $7 \cdot 24$. Phonation occurred in 13 per cent. The pupils were five millimetres in 15 cases, four millimetres in 43 cases, three millimetres in 24 cases, about two millimetres in four cases, and normal in 13 cases. One case was not recorded. There was no cyanosis in 97 cases. The three showing cyanosis are as follows :-

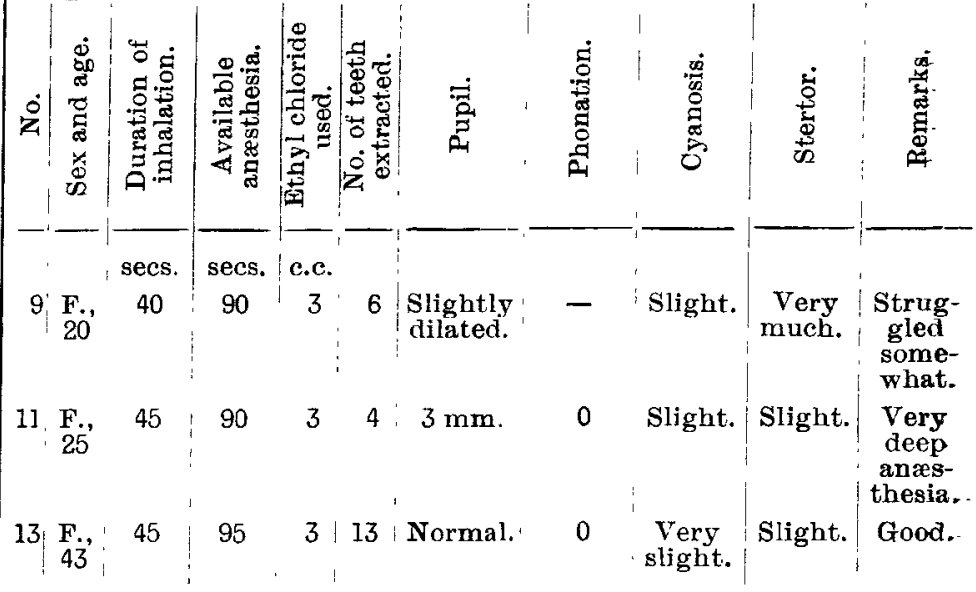

Stertor occurred in 19 per cent. of the cases. 15 occurred in females; of these one is recorded above under cyanosis, one is noted as "very much," and the rest were "slight," "very slight," or "yes." In the four males two are recorded as "slight," one as "very slight," and the character of one is not noted.

In a number of cases all through these records there is nothing noted under "remarks" and 1 think I am quite justified in including these under such headings as satisfactory, good, or very good, because any slight deviation from the normal was at once noted.

Involuntary micturition occurred in 4 per cent. All were females; one was a child, aged nine years, and the rest were adults. 5 per cent., all females, vomited on recovery. As much importance is attached to this event $I$ am giving the notes, leaving out the sex.

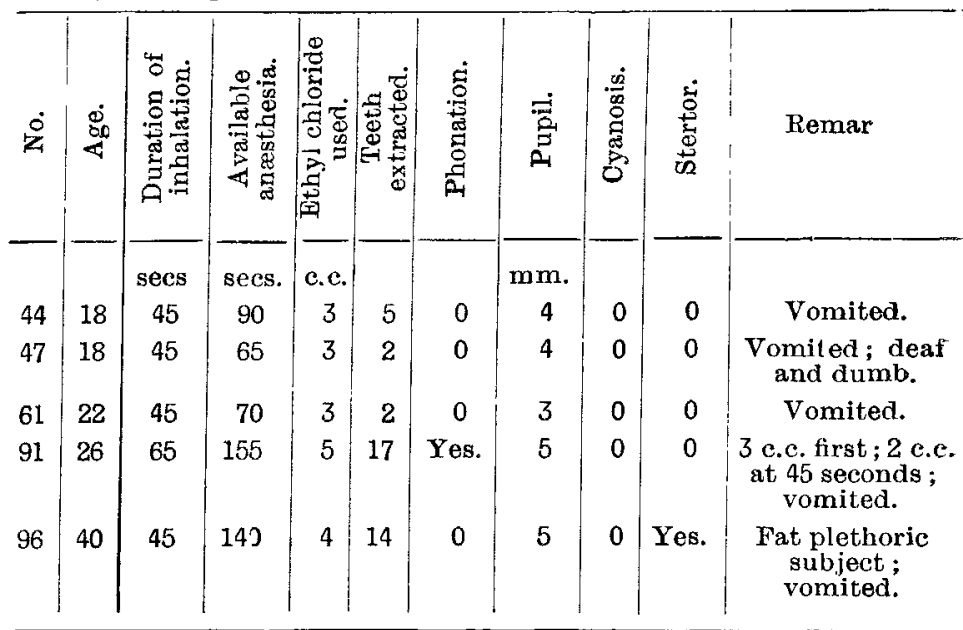

It will be seen that one was a case of re-inhalation and one was a fat plethoric subject. With regard to struggling 
and excitement on going under there were three cases and on recovery four cases. There was one case of hysterical excitement on recovery. There were four cases of slight movement on recovery but omplete analgesia; also one case in which there was movement whilst deeply under. The various pathological conditions noted are as follows : epileptic with enlarged tonsils; specific cirrhosis of the liver; anæmia and faint before inhalation (the colour was improved and the patient said he felt better after operation); lips blue and cold extremities before; alveolar abscess; tuberculous knee; anæmia (four cases in all); cardiac disease; and syphilis. Exception should, I think, be made to the note of one case-viz., that of No. 4, a man, aged 30 years. Induction occurred in 60 seconds. Anæsthesia lasted 60 seconds, with three cubic centimetres; 13 teeth were extracted. "Analgesia was complete but not anæs. thesia." I think this remark must be in error, as 60 seconds is given as available anæsthesia ; there was stertor, although very slight; and, further, in my experience it is improbable that 13 teeth could be extracted under an analgesic condition without movement or struggling.

Somnoform alone.-This 100 records comprise 76 females and 24 males. 'The oldest was 52 years and the youngest was six years. The average age was 20.48 years. With regard to the duration of induction, the longest was 120 seconds. This case was a female, aged 15 years; the dose given was three and a half cubic centimetres. Anæsthesia was induced in 65 seconds. 16 teeth were extracted. There were phonation and stertor. The pupils were four millimetres. There was no cyanosis. The patient was very nervous and held her breath during induction. The shortest time of induction was 25 seconds. There were two cases. Case 67 was a male, aged nine years. The dose given was one and a half cubic centimetres. Available anæsthesia. 50 seconds. Three teeth were extracted. The pupils were four millimetres. There was no phonation, \&c. Cuse 98 was a female, aged five vears. Anæsthesia was induced in 25 seconds. Duration of anæsthesia, 55 seconds; the dose given was two cubic centionetres. Five teeth were extracted. The pupils were normal. There was no phonation, \&c. The average time was $41 \cdot 3$ seconds. With regard to the dose, the largest was five cubic centimetres in one case (No. 93). The patient was a female, aged 30 years. Induction period, 65 seconds and anæsthesia lasted 85 seconds. The teeth extracted numbered 19. There was phonation. The pupils were four millimetres. There was no cyanosis or stertor. The smallest dose was one and a half cubic centimetres (three cases). Case $6 \hat{0}$ was a female, aged 11 years. Induction period, 30 seconds and anæsthesia lasted 70 seconds. Four teeth were extracted. The pupils were four millimetres. There was no phonation, \&c. Case 67 was a male, aged nine years. Induction period, 25 seconds and anæsthesia lasted 50 seconds. Three teeth were extracted. The pupils were four millimetres. There was no phonation, scc. Case 69 was a female, aged 14 years. Induction period, 30 seconds and anæsthesia lasted 60 seconds. Two teeth were extracted. The pupils were normal. There was no phonation, \&c. The average dose was 2.77 eubic centimetres for the whole series.

Availablc ancesthesia.-No re-innalation was given in this series. The longest time was 180 seconds, Case 62, a female, aged 15 years. Induction period, 35 seconds; the dose administered was three cubic centimetres. The teeth extracted numbered 15. The pupils were five millimetres. There was slight stertor. There was no phonation or cyanosir. The shortest time was ten seconds, Case 51, a male, aged ten years. Induction period, 30 seconds; the dose adininistered was two and a half cubic centimetres. The teeth extracted numbered three. The pupils were normal. There was no phonation, \&c. Average time, $64 \cdot 88$ seconds. The greatest number of teeth extracted from one case was 24 , Case 16, a female, aged 27 years. Induction period, 45 seconds; the dose administered was three cubic centimetres. Anæathesia lasted 105 seconds. The pupils were five millimetres. There was no phonation, stertor, or cyanosis. No remarks were noted. One tooth was extracted in one case, No. 63, a female, aged 33 years. Induction period, 30 seconds. The dose administered was two and a half cubic centimetres. Anæsthesia lasted 80 seconds. The pupils were normal. There was no phonation, \&c. The average number of teeth extracted was 666 . Phonation was recorded in 13 per cent. The pupils were five callimetres in 15 per cent., four millimetres in 41 per cent., three millimetres in 3 per cent., and normal in 41 per cent. No cyanosis was recorded. Stertor occurred in 10 per cent. of the cases. The actual notes are: "Yes" in six; "slight" in three; and "distinct stertor" in one. The remarks are as follows. Excitement on recovery, six; struggling on recovery, two; excitement during induction, one; poor anæsthesia, excitement and swearing, a male, aged 35 years (time not noted), one; total, 10 per cent. There was one case of vomiting afterwards, Case 3, a male, aged 14 years. Induction period, 35 seconds and anæsthesia lasted 50 seconds. The dose administered was two and a half cubic centimetres. The number of teeth extracted was three. The pupils were four millimetres. There was no phonation, cyanosis, or stertor. There was one case of involuntary micturition, Case 15, a male, aged 29 years. Of abrormally long analgesia after there were two cases. I give notes of one, Case 8, a female, aged 15 years. Induction period, 35 seconds and anæsthesia lasted 65 seconds. The dose administered was two and a half cubic centi. metres. The number of teeth extracted was seven. The pupils were three millimetres. There was no phonation, stertor, or cyanosis. There were 65 seconds of analgesia after. There were three cases of vertical nys'agmus, three very nervous cases, two very obese females, four cases of holding of the breath during inhalation, and one case of muscular spasms. This was Case 81 , a male, aged 37 years. Induction period, 60 seconds and anæsthesia lasted 60 seconds. The dose administered was three cubic centimetres. The number of teeth extracted was four. The pupils were normal. There was no phonation, cyanosis, or stertor. He had undergone a recent mastoid operation. One case, a child, had slightly occluded upper air passages and a breath of air was given at 20 seconds. There were one case of very sudden recovery and one suffering from extreme anæmia.

Nitrous oxide and chloride of ethyl.-The inhalers employed in this mixture and the following are as follows: 1 . That of my own as described above and used in the following way. To the tap is affixed the rubber tube connected to the nitrous oxide cylinders. The measure glass is now charged and placed near at hand. The gas is slightly turned on with the foot-key. The facepiece is carefully adjusted during an inspiration. When the bag is almost full of gas the tube is disconnected and replaced by the charged measure. After a few breaths the fluid is permitted to evaporate in the way described above only rather more quickly. 2. A Bath's twoway tap gas apparatus, the gas and fluid being introduced by a special opening just above the bag, the principle of which was described by me in $1903^{\mathrm{l}}$ The advantage of this apparatus is that the bag can be filled witn gas before the inhaler is placed to the patient's face, but it has been found that the evaporation is not quite so satisfactory if the bag is wet inside. This inhaler was also described in $1904 .^{2}$ 3. Dr Hewitt's special gas apparatus and method. This was only used once. It will be seen that the measure glass was used in all administrations. In the majority of cases the amount of gas given was one gallon.

There were 80 females and 20 males. The oldest was 60 years of age and the youngest were two at the age of nine years. The average age was $25 \cdot 50$ years. With the object of determining the bent length of time to exhibit nitrous oxide as a preliminary to chloride of ethyl various times were given and I regret to find on going through these records that the number of administrations do not correspond. Previously to these records were a number in which Dr. Hewitt's apparatus and method were tried and I think the reason for not giving a larger number of administrations with more gas than one gallon was that it had been found that better results were attained by using less and a small bag and introducing the chloride of ethyl quite early in the administration.

Class A.-There were seven cases in which nitrous oxide was re-breathed for from 20 to 30 seconds before the chloride of ethyl was introduced and the mixture for about 47 seconds. The dose of chloride of ethyl was three cubic centimetres. The time taken to produce anæsthesia was about 102 seconds and an available period of anx-tbeia a little over 60 seconds. 46 teeth were extracted in the seven cases. There was phonation on going under in three cases. The pupils were normal in one case, four millimetres in five cases, and five millimetres in one case. Cyanosis occurred in one 
case and a breath of air was given. In another case a breath of air was given at 40 seconds. No stertor was recorded. In Case 1 between one and two gallons of gas were given. There was no vomiting in any case.

Class B.-There were 41 cases in which nitrous oxide was re-breathed for 15 seconds previously to the introduction of chloride of ethyl. The mixture was given for about 50 seconds. The average time of induction was a little over 66 seconds, the longest being 105 seconds and the shortest 40 seconds. The average available period of anæsthesia was a little over 68 seconds. The longest time was 120 seconds but was not a satisfactory anæsthesia, case 45 , a female, aged 22 years ; the mixture, 90 seconds, dose three cubic centimetres. 18 teeth were extracted. There was phonation. The pupils were four millimetres and there were stertor and cyanosis. The patient was given a breath of air at 50 seconds. There were much excitement and resistance on recovery but complete analgesia. The shortert time was 35 seconds, Case 73 , a male, aged nine jears. The mixture 25 seconds. The dose administered was three cubic centimetres. Two teeth were extracted. The pupils were four millimetres. There was no phonation but slight stertor and cyanosis. The average dose was a fraction under three cubic centimetres, practically three cubic centimetres. The largest was five cubic centimetres, giıing 70 seconds. The smallest was one and a half cubic centimetres, three cases, producing anæsthesia of 60,55 , and 42 seconds respectively. Phonation occurred in 13 cases, one on going under and the others were not noted as to when. The pupils were five millimetres in 11 cases ; four millimetres in 18 cases; three millimetres in six cases; and normal in six cases. Cyanosis was "slight" or "very slight" in 13 cases and "yes" in ten cases. Stertor was noted in nine cases as " jes" and "slight" or "rery slight" in four cases. One breath of air was given at 50 seconds in two cases; one breath at 40 seconds in one feeble and neurotic patient; and one breath at 35 seconds in one case (Hewitt's apparatus). One breath of air was given at a time not recorded in one case. There was excitement on going under in one case. There were excitement and resistance on recovery but analgesia in three cases. No case of vomiting and no case of involuntary micturition. There was one case of vertical nystagmus. With regard to pathological conditions, there was one case each of exophthalmic goitre, epithelioma of the cheek, and anæmia.

Class $C$.- There were 51 cases in which nitrous oxide was re-breathed for ten seconds previously to the introduction of chloride of ethyl and the mixture for akout 52 seconds. The oldest patient was 66 years of age and the youngest was nine years of age. The average time of induction was 62 seconds. The longest time was 100 seconds, Case 12, a female, aged 24 years. Gas was given for ten seconds and the mixture for 90 seconds. Anæsthesia lasted 80 seconds The dose administered was three cubic centimetres. 'The number of teeth extracted was nine. There was ptonation. The pupils were normal. There was slight cyanosis but no stertor. The shortest time was 30 seconds, Case 56, a male, aged nine years. Gas was given for ten seconds and the mixture for 20 seconds. Anæsthesia lasted 40 seconds. The number of teeth extracted was two. The pupils were normal. There was no phonation, \&c. The average dose of chloride of ethyl was 3.48 cubic centimetres ; the largest was five cubic centimetres. Cases 40 and 88 below are given as examples as there are several (second inhalations not counted). The smallest dose was two cubic centimetres, Case 14. The greatest number of teeth extracted was 15 , Case 82 , a female, aged 37 years. The total induction was 85 seconds, the anæsthesia was for 100 seconds, and the dose was four cubic centimetres. The pupils were normal. There was no phonation, cyanosis, or stertor. The patient was anæmic; it was a typical, good case. The smallest number of teeth extracted was two, of which there were several instances. Case 14 below is a good example. The average available anæsthesia for this class was 71 seconds. The longest period was 120 seconds (two cases). Case 40, a female, aged 50 years, had the mixture for 65 seconds. The dose administered was five cubic centimetres. The number of teeth extracted was 12 . The pupils were five millimetres. There was no phonation, \&c. The patient was anæmic. In Case 88, a female, aged 19 years, the mixture was given for 55 seconds. The dose administered was five cubic centimetres. The number of teeth extracted was 15 . There was no phonation. The pupils were five millimetres. There was cyanosis. Stertor was slight. Oase No. 61 is of interest, as it shows the longest anæsthesia for three cubic centimetres. The patient was a female, aged 23 years. The mixture was given for 35 seconds. Anæsthesia lasted 100 seconds. The number of teeth extracted was six. There was no phonation or cyanosis. The pupils were four millimetres. Stertor was slight. The patient was deaf and had the frontal sinus opened recently. There was reported excitement under the anæsthetic but she was quite quiet under this mixture. The shortest. time was 35 seconds, Case 14, a female, aged 23 years. The mixture was given for 75 seconds. The dose administered was two cubic centimetres. The number of teeth extracted was two. There was phonation. The pupils were normal. Cyanosis was slight. There was no stertor. In Case 30 an extra dose of chloride of ethyl was given but the result of the first dose only is added to the sum of average available anæsthesia, \&c. The full record is now given. The patient was a male, aged 21 years. Gas was given for ten scconds and the mixture for 55 seconds. The dose administered was three cubic centimetres. Anæsthesia lasted 55 seconds. A further dose of five cubic centimetres for 40 seconds gave a further available anæsthesia of 150 seconds. The number of teeth extracted was 19. The re was phonation. The pupils were four millimetres. There were stertor and cyanosis ; also marked muscular rigidity all through. Case 31 was omitted in this series because the tongue fell back, thus temporarily obstructing respiration at 40 seconds. There were 11 cases of phonation. The pupils were five millimetres in 15 cases, four millimetres in 26 cases, and normal in ten cases. There was slight cyanosis in 32 cases, and "very slight" in one case. Stertor without further remark was present in six cases, "slight" in 13 cases, and "very 'slight" in one case. Involuntary micturition occurred in one case and prolonged recovery in two cases of pulmonary tuberculosis. Two cases went under quietly but had violent struggling on recovery. There were great excitement during administration in one case, struggling on recovery in one case, and excitement on recovery in one case. There was no vomiting. The pathological conditions other than mentioned above were a very large abscess in the neck, chronic Bright's disease, and an anæmic woman.

Nit"ous uxide and somnoform.-This series is also divided into classes $\mathrm{A}, \mathrm{B}$, and $\mathrm{C}$. One gallon of nitrous oxide was giren in all cases.

Class $A$.- Two cases which I am unable to include in $B$ or $C$. Case 7, a female, aged 19 jears. Nitrous oxide was given for 20 seconds. The mixture was given for 50 seconds. The whole induction period was 70 seconds. Available anæssthesia lasted 120 seconds. The dose administered was three cubic centimetres. The numker of teeth extracted was seven. The pupils were five millimetres. There was no phonation, cjanosis, or stertor. The patient vomited afterwards. Case 9 was a female, aged 12 jears. Nitrous oxice was given for five seconds. The mixture was given for 30 seconds. The whole induction period was 35 seconds. Available anæsthesia lasted 40 seconds. The dose administered was three cubic centimetres. One tooth was extracted. The pupils were normal. There was no phonation, \&c. There was no reinhalation in this series of 100 .

Class B.-There were 72 cases in which nitrous oxide was re-breathed for 15 seconds before the somnoform was given in mixture. The oldest patient was 42 years and the youngest was eight years. The arerage time during which the mixture was given was 34 seconds. The average duration of induction was 49 seconds. The longest time was 60 seconds and the shortest time was 30 seconds. The average available anæsthesia was 78 seconds. The longest period of anæsthesia was 175 seconds, Case 60, a male, aged 21 years. Induction period 60 seconds. The dose administered was four cubic centimetres. The number of teeth extracted was nine. There was no phonation or stertor. The pupils were four millimetres. There was slight cyanosis. This patient held his breath during inhalation and there was involuntary micturition. The shortest time was 30 seconds, Case 50 aged 17 years. Induction period, 40 seconds. The dose adwinistered was three and a half cubic centimetres. The number of teeth extracted was three. The pupils were normal. There was no phonation, \&c. The average amount of somnoform used was a fraction over three cubic centimetres. The largest amount was five cubic centimetres, given on four occasions. One case, No. 30, was a male, aged 16 years. Induction period, 45 seconds. Anæsthesia lasted 90 seconds. The number of teeth extracted was 12 There was no phonation, cyanosis, or stertor. The pupils 
were four millimetres. There was no nystagmus. The smallest amount was one and a half cubic centimetres, Case 39, aged 23 years. Induction period, 45 seconds. Anæsthesia lasted 75 seconds. The number of teeth extracted was six. There was phonation. The pupils were three millimetres. There was no cyanosis or stertor. Phonation occurred in 14 cases, the pupils were five millimetres in eight cases, four millimetres in 29 cases, three millimetres in two cases, and normal in 33 cases. Cyanosis was "slight" in four and noted as "yes" in three cases. Stertor was recorded in six cases. The greatest number of teeth extracted was 24 . Case 12, a female, aged 19 years. Induction period, 55 seconds. Anæsthesia lasted 80 seconds. The dose administered was five cubic centimetres. The pupils were four millimetres. There was no phonation, \&c. One tooth was extracted on five occasions. Involuntary micturition occurred in four cases, vomiting as well in one of those, excitement on going under in one case, hysterical attack before in one case, exeitement or struggling after in two cases, great excitement on recovery in one case, struggling (not stated when) in one case (strong athletic man), and nystagmus in three cases. There were a large alveolar abscess and a stiff jaw in two cases. One patient was a very plethoric woman who had lupus and one was a patient with pulmonary tuberculosis.

Class C.-There were 26 cases in which nitrous oxide was re-breathed for ten seconds before the somnoform was given in mixture. The average time during which the mixture was given was about 36 seconds. The oldest patient was 37 years of age and the youngest was 13 years. The average time of induction was a little over 46 seconds. The longest time was 60 seconds and the shortest time was 40 seconds. The available andsthesia was for 75 seconds. The longest was for 150 seconds. Case 78, a female, aged 34 years; induction period 45 seconds. The dose administered was four cubic centimetres. The number of teeth extracted was ten. The pupils were normal. There was no cyanosis, \&c. The shortest time was 15 seconds, Cuse 75 , a female, aged 34 years; period of induction, 50 seconds. The dose administered was three cubic centimetres. The number of teeth extracted was two. The pupils were normal. There weie no phonation and no cyanosis. There was slight stertor. Two students extracted. The dose of somnoform was four cubic centimetres in seven cases, five cubic centimetres in one case, and two and a half cubic centimetres in one case. The rest were three cubic centimetres. The greatest number of teeth extracted from one patient was 26 in 90 seconds, Case No. 73 , a female, aged 19 years; induction period, 45 seconds. The dose administered was five cubic centimetres. The pupils were three millimetres. There were slight phonation and stertor. There was no cyanosis. One tooth was extracted in one case. There was phonation in ten cases. The pupils were five millimetres in one case, four millimetres in eight cases, three millimetres in four cases, and normal in 13 cases. There were cyanosis in four cases, all slight, and stertor in five cases, all slight. There was loud phonation all through but anæithesia was complete in two cases. Nystagmus occurred in one case, involuntary micturition in one case retching in one case, slight retching in two cases, and anæmia with headache in one case.

Space will not permit of my publishing a more complete analysis and $I$ have avoided as far as possible long lists. It should be remembered that many patients had to wait for a long time before their turn came in an adjoining room where the noises of those being operated on could be clearly heard. This will help to explain to some extent the excitement on going under and also on recovery. Further, there is no lavatory accommodation in connexion with this waiting room. The proportion of cases of involuntary micturition might possibly have been less were conditions otherwise. In some cases the operative procedure was continued after the patient manifested signs of recovery. In such cases I generally made inquiries after complete recovery and in almost every case was told that no pain was felt. The analgesia was complete but such a procedure determined excitement and struggling. It was not unusual for such patients to state that they had an unpleasant dream. Moreover, operating on a patient recovering-i.e., when the swallowing reflex is present-will, of course, tend to increase vomiting. All the cases were unprepared but from the hour of the day it is concluded that the majority had not taken food for some time. In the case of somnoform it should be noted that it was not given in cases of advanced cardiac disease or in cases in which trouble might be anticipated. It is not, however, to be understood that they were selected cases. It is remarkable to note the length of time nitrous oxide can be re-breathed in a one-gallon bag in the presence of chloride of ethyl without producing cyanosis or stertor. Case 20 is an instance. The patient was a female, aged 17 years. Gas was given for 15 seconds and the mixture for 90 seconds, total 105 seconds. Anæsthesia lasted 60 seconds. The dose of ethyl chloride administered was three cubic centimetres. Three teeth were extracted. There was no phonation. The pupils were five millimetres. There were no cyanosis and no stertor.

\section{A CASE OF PREGNANCY COMPLICATED BY CHOREA GRAVIDARUM AND ECLAMPSIA; RECOVERY.}

By H, U. GOULD, M.B., B.C. CANTAB., M.R.C.S. ENG., L.R.C.P. LOND,

AND

O. M. HINDS HOWELL, M.B., B.CH. OXON., M.R.O.S. ENG., M.R.C.P.LOND.

THE notes of the following case are of interest owing to the fact that the albuminuria and subsequent eclampsia appeared whilst the patient was under observation in St. Bartholomew's Hospital where she had been admitted suffering from chorea.

The patient, aged 19 years, a primigravida, first came under observation on Oct. 11th, 1904. At that time she applied to the obstetrical out-patient department for a maternity letter. She was then noticed to have choreic movememts in the left arm and paresis in that arm and the left leg. On the 20th she was admitted to the hospital under the care of Sir Dyce Duckworth. The following history was obtained. She had been married nine months previously and was subsequently deserted by her husband. She now lived with her father and stepmother. Five weeks before admission after a fright caused by a fire occurring in her room, she began to exhibit choreic movements in the left arm and hand. The condition had gradually become more severe, subsequently involving the left side of the face and the left leg. The last catamenial period occurred from Feb. 23rd to 27th-i.e., 33 weeks previously. There was a history of rheumatism on the father's side but the patient previously had exhibited no rheumatic tendency.

On examination the lungs were found to be natural ; a slight murmur, probably hæmic, was heard at the pulmonary base, otherwise the heart appeared to be unaffected. There were choreic movements of the left arm and distinct paresis of that limb. The patient was unable to pick up a pin or to feed herself with that arm. The left side of the face and the left leg were similarly, but more slightly, affected. There were present the ordinary signs of pregnancy at the end of the eighth month. The position of the child was ascertained to be left occipito-anterior; the fotal heart was counted at 152. The knee-jerks and ankle-jerks were brisk; a flexor plantar response was obtained. There was no odema of the legs. The urine was acid, of specific gravity 1020 , and contained no sugar. There was a slight trace of albumin which cleared up in the course of a few days and was then entirely absent for over a month. The temperature was $98 \cdot 8^{\circ} \mathrm{F}$., the pulse was 80 , and the respirations were 22 . The patient was confined to bed and was given 15 grains of sodium bromide twice daily.

On Oct. 24th the patient's condition was improved and she was able to wash herself with her left arm. On the 31st there was still further improvement and she could use her left arm fairly well. She was allowed to get up. The bromide was discontinued. On Nov. 21st choreic movements were absent and there was good power in the left arm. The urine was quite free from albumin. On the $28 \mathrm{th}$ a thick cloud of albumin was found in the urine. On Dec. 2nd albumin was increasing in the urine $(0.3$ per cent.) The patient complained of headache and abdominal pain at the level of the fundus uteri. There was no vomiting. (This symptom was absent throughout.) At 8.30 P.M. she had an eclamptic fit with general convulsions lasting for ten minutes. The temperature was $99 \cdot 2^{\circ}$, the pulse was 